\title{
Minimum Quench Energy and Early Quench Development in NbTi Superconducting Strands
}

\author{
M. Breschi, L. Trevisani, M. Boselli, L. Bottura, A. Devred, P. L. Ribani, and F. Trillaud
}

\begin{abstract}
The stability of superconducting wires is a crucial task in the design of safe and reliable superconducting magnets. These magnets are prone to premature quenches due to local releases of energy. In order to simulate these energy disturbances, various heater technologies have been developed, such as coated tips, graphite pastes, and inductive coils. The experiments studied in the present work have been performed using a single-mode diode laser with an optical fiber to illuminate the superconducting strand surface. Minimum quench energies and voltage traces at different magnetic flux densities and transport currents have been measured on an LHC-type, $\mathrm{Cu} / \mathrm{NbTi}$ wire bathed in pool boiling helium I. This paper deals with the numerical analysis of the experimental data. In particular, a coupled electromagnetic and thermal model has been developed to study quench development and propagation, focusing on the influence of heat exchange with liquid helium.
\end{abstract}

Index Terms-Heat transfer, NbTi, pool boiling helium, superconducting wire, thermo-electrical stability.

\section{INTRODUCTION}

$\mathbf{T}$ HE latest developments in magnet technology for particle accelerators and fusion magnets push towards the design and manufacturing of superconducting magnets with the highest possible magnetic fields. In these conditions, low temperature superconductors work at the limit of their current-carrying capabilities. Any small energetic disturbance may be able to drive the entire magnet normal due to the development of quenches. In order to reproduce in a laboratory experiment energy disturbances similar to those occurring in the real magnet operation, several kinds of heaters have been used [1], [2]. A new heater technology has been developed at CEA/Saclay based on commercial diode lasers. The measurement technique and the details of the experimental setup have been described elsewhere [3], [4]. This paper deals with the numerical analysis of the experimental data obtained at CEA/Saclay in pool boiling helium I. A coupled electromagnetic and thermal model has been developed to simulate the resistive transition of the superconducting

Manuscript received August 28, 2006.

M. Breschi, L. Trevisani, M. Boselli and P. L. Ribani are with the Department of Electrical Engineering, University of Bologna, Italy (e-mail: marco.breschi@unibo.it; luca.trevisani@mail.ing.unibo.it; marco. boselli@studio.unibo.it; pierluigi.ribani@unibo.it).

L. Bottura is with CERN, AT/MTM, Switzerland (e-mail: luca.bottura@ cern. ch).

A. Devred is with CEA/Saclay, DSM/DAPNIA/SACM, France, and with CERN, AT/MAS, Switzerland (e-mail: arnaud.devred@cern.ch).

F. Trillaud is with the Massachussetts Institute of Technology, Cambridge, MA 02139 USA (e-mail: trillaud@mit.edu).

Digital Object Identifier 10.1109/TASC.2007.898373

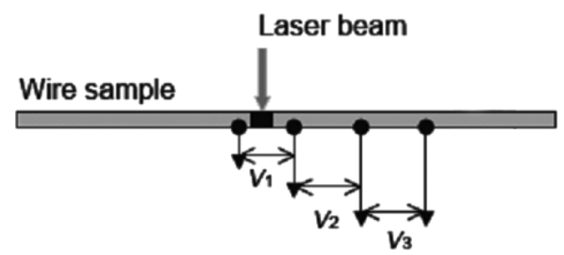

Fig. 1. Schematics of the location of the voltage taps.

multifilament composite wire, with a particular emphasis on the description of the heat exchange towards liquid helium.

\section{EXPERIMENTAL FACILITY}

The measurements were performed on an LHC-type (02), $\mathrm{Cu} / \mathrm{NbTi}$ wire with a $0.825-\mathrm{mm}$ diameter and a $1.95 \mathrm{Cu} / \mathrm{NbTi}$ ratio. The experiments were carried out at different transport currents (between $80 \%$ and $95 \%$ of the wire critical current) and background magnetic flux densities (from 5 to $7 \mathrm{~T}$ ). The wire critical current was measured in situ to be $320 \mathrm{~A}$ at $7 \mathrm{~T}$ and $545 \mathrm{~A}$ at $5 \mathrm{~T}$. The active sample length was $8 \mathrm{~cm}$. To induce thermal disturbances, the wire was illuminated with a single-mode diode laser over a $0.8-\mathrm{mm}$ region centered in its middle section, with pulse length varied between $20 \mu \mathrm{s}$ and $2 \mathrm{~ms}$. The voltages that developed along the sample were measured through a set of voltage taps illustrated in Fig. 1.

\section{MODEL DESCRIPTION}

The study reported in the present work is based on a coupled thermo-electromagnetic 1D model of the wire sample [5]. The coupling is realized through temperature-dependent electrical properties of materials and the introduction in the thermal model of the Joule power calculated with the electromagnetic model. The wire is divided into $N_{\mathrm{sec}}$ sectors, allowed to have different lengths.

\section{A. Electromagnetic Model}

In each sector, the superconducting filaments are lumped into a single electrical component describing the superconducting part of the strand. A different component is introduced in parallel to describe the non-superconducting part of the wire cross section. The non-linear voltage-current characteristics of the superconductor is represented by the customary power law, whose exponent is the so-called $N$-value. In the simulations, we used $N$-values derived from in situ measurements at different fields [4]. As critical current measurements were only available at 4.2 K, a number of critical surface parameters were set to typical values for $\mathrm{NbTi}$ wires. The Joule power calculated in the model 
accounts for the dissipation in the superconducting and resistive materials.

\section{B. Thermal Model}

In the thermal model, each wire sector is allowed to exchange heat with the neighboring sectors through conduction along the wire, and with the helium by the usual cooling mechanisms. The main heat source is the Joule power dissipated in the wire due to the transport current. A non uniform mesh with coarser elements at the strand ends and finer elements around the heated region $(0.2 \mathrm{~mm})$ has been used. Only four sectors, located near the sample middle position are subjected to the external heat disturbance simulating the laser illumination. The energy balance ordinary differential equations for a given sector can be written as

$$
\begin{aligned}
m_{i} c_{p}\left(T_{i}\right) & \frac{d T_{i}}{d t}=p_{J, i}+p_{e x t, i} \\
& +k\left(T_{i}\right) S\left(\frac{T_{i+1}-T_{i}}{\Delta l_{i, i+1}}+\frac{T_{i-1}-T_{i}}{\Delta l_{i-i, i}}\right)-p_{H e, i}
\end{aligned}
$$

where $m_{i}, c_{p}$, and $T_{i}$ are the mass, the specific heat at constant pressure and the temperature of sector $i, p_{J, i}$ is the Joule power dissipated in sector $i, p_{\text {ext }, i}$ is the thermal power deposited onto sector $i$ by the laser illumination, $S$ is the wire cross-sectional area, $\Delta l_{i, j}$ is the distance between the middle points of sectors $i$ and $j$ and $T_{H e}$ is the helium bath temperature. The wire thermal conductivity, $k$, is computed as a weighted average of the thermal conductivities of the superconducting and non-superconducting components, which are both temperature-dependent. The heat $p_{H e, i}$ transferred to the helium bath by sector $i$, is given by:

$$
p_{H e, i}=h_{i}\left(T_{i}, T_{H e}\right) S_{i}^{w e t}\left(T_{i}-T_{H e}\right)
$$

where $S_{i}^{\text {wet }}$ is the wetted surface of sector $i$, and $h_{i}$ is a heat exchange factor evaluated as a weighted average between the linear coefficient obtained in film boiling and the nonlinear coefficient obtained in nucleate boiling, as explained in the following sections.

\section{Heat Exchange With Liquid Helium}

The theoretical evaluation of the heat exchange coefficient with liquid helium represents a complex task. This coefficient depends on several parameters, which are often unknown and difficult to measure. To simplify, two different phases are distinguished in the helium boiling process close to a heated metallic surface. In a first phase (low heat flux), called nucleate boiling, vapor bubbles appear on the surface of the wire; the location of their nucleation sites is almost unpredictable and the different bubbles are separated from each other. In a second phase (higher heat flux), an evaporated helium thin film forms close to the refrigerated surface. This phase is referred to as film boiling, and is characterized by a heat exchange coefficient much lower than in the previous phase, due to a thermal insulation effect caused by the vapor film between the surface and the liquid helium. Numerous experimental data are available for transient nucleate-boiling heat-transfer coefficients, which present a large scatter as a function of surface material, surface roughness, surface condition (clean, oxidized, coated, etc.). In our model, we assume that the heat exchange process during transient nucleate boiling is mainly governed by the Kapitza thermal resistance [6] described as

$$
h_{n u c l, i}=\frac{Q_{K a p}}{S_{i}^{\text {wet }}\left(T_{i}-T_{H e}\right)}=\frac{A\left(T_{s}^{m}-T_{H e}^{m}\right)}{S_{i}^{\text {wet }}\left(T_{i}-T_{H e}\right)}
$$

where $Q_{K a p}$ is the Kapitza thermal flux. The values used for the parameters $A$ and $m$ in (2) are $A=242 \mathrm{~W} / \mathrm{m}^{2} \mathrm{Km}$ and $m=$ 2.8. These values are in the range of those indicated in [8] and have been selected in order to have a best fit of the experimental results.

Beyond the critical heat flux, the formation of an insulating gas film on the wire surface establishes the film boiling regime. A relevant aspect in the modeling is the evaluation of the maximum heat flux at which the sudden drop of heat exchange coefficient occurs, i.e., the transition from nucleate to film boiling. In the literature, various theoretical or experimental models are available which correlate the onset time of film boiling $\left(t_{o n}\right)$ with the total energy flux transferred to the helium up to film boiling formation, $E_{l i m}$ [6], [7], [9]. In this work, in order to extend these relations to the case of variable heat fluxes, we consider the following correlation between time and energy

$$
E_{\lim }=\alpha t_{o n}^{n}
$$

where $\alpha$ is a constant coefficient. This correlation is equivalent to the original one when the heat flux is constant. In our computations, we use: $\alpha=720 \mathrm{~J} / \mathrm{m}^{2} \mathrm{sn}$ and $n=0.6$. After $E_{\text {lim }}$ is reached, the heat transfer coefficient drops to its film boiling value, $h_{\text {film }}$, taken equal to $250 \mathrm{~W} / \mathrm{m}^{2} \mathrm{~K}$. Such value is compatible with most measurements on copper wires.

Several steady-state experiments, realized with a constant heat flux towards the helium, have shown that, below a certain heat flux value, or below a given threshold, $\Delta T$, film boiling does not occur even after a long time. This is due to the possibility for the deposited and/or generated heat to be removed by nucleate boiling without forming a gas film [6]. Typical $\Delta T$ values range from 0.6 to $0.8 \mathrm{~K}$ and here, we use $0.7 \mathrm{~K}$. In our model, the numerical integration of the energy flux transferred to the helium by a wire sector only starts after the sector temperature reaches $4.9 \mathrm{~K}$, corresponding to $\Delta T=0.7 \mathrm{~K}$, while the helium bath is kept at a constant temperature of $4.2 \mathrm{~K}$. When the integrated energy flux equals the limit defined in (3), the sector enters the film-boiling regime. However, to prevent numerical instabilities, this transition is not modeled as an abrupt change in local heat transfer coefficient of a single sector. Indeed, the gas film formation is a collective phenomenon rather than a local transition. In the present work, a Gaussian smearing of the heat transfer coefficient has been implemented along the sample length. The smearing is applied by defining for every sector the following discrete function: $H_{\text {check }, i}=0$ for nucleate boiling and $H_{c h e c k, i}=1$ for film boiling, with $i=1$, 
$N_{\text {sec }}$. The smeared value of the heat transfer coefficient is given by

$$
H_{\text {smeared }, i}=\frac{\sum_{j=1}^{N s e c} w_{i, j} H_{\text {check }, j}}{\sum_{j=1}^{N s e c} w_{i, j}}
$$

where the weight functions $w_{i, j}$ are defined as

$$
w_{i, j}=\frac{1}{\sqrt{2 \pi} \sigma} \int_{x_{l, j}}^{x_{r, j}} \exp \left\{-\frac{\left[x_{m, i}-x\right]^{2}}{2 \sigma^{2}}\right\} d x
$$

Here $x_{l}, j, x_{r}, j$, and $x_{m, i}$ represent the left and right borders of element $j$ and the middle point of element $i$. The shape of the smearing region is controlled by $\sigma$, which is taken equal to $1 \mathrm{~mm}$. A parametric study as a function of $\sigma$ has shown that values larger or equal to $1 \mathrm{~mm}$ guarantee good convergence and numerical stability, with little influence on the simulation results, while lower values lead to nucleate-to-film boiling transitions that are too abrupt.

In summary, for each sector, the heat transfer coefficient is computed as

$$
\begin{array}{r}
h_{i}=\left[1-H_{\text {smeared }, i}\right] h_{\text {nucl }, i}+H_{\text {smeared }, i} h_{\text {film }} \\
\text { with } i=1, N_{\text {sec }}
\end{array}
$$

This definition shows that the value of $h$ for each sector is affected by the values of $h$ in the neighboring sectors, allowing for a smooth transition in time and space from nucleate to film boiling.

An additional feature of the model is the possibility for a wire sector to enter the film boiling regime, and, subsequently, to recover the nucleate boiling regime if the temperature decreases. In our parameterization, this backward transition occurs when the sector temperature decreases below $4.9 \mathrm{~K}$, consistently with the previous considerations about the nucleate-to-film-boiling transition. This feature of the model is essential to accurately describe the recoveries at long times, when maintaining the film boiling regime leads to an artificially low value of heat transfer coefficient.

\section{Simulation Results}

\section{A. Voltage Traces}

The procedures described in the previous sections have been applied to analyse a wide set of experimental data obtained for the $\mathrm{LHC} \mathrm{Cu} / \mathrm{NbTi}$ wire sample described above. Measurements performed in 5, 6, and 7-T background field have been simulated, with transport currents in the range from 80 to $95 \%$ of the wire critical current. The voltage traces recorded at three different locations (see Fig. 1) along the wire sample are used for the comparison between measurements and numerical simulations. The voltage traces for $I_{\mathrm{op}}=85 \% I_{\mathrm{C}}$ at $6 \mathrm{~T}$ are reported in Fig. 2 for three different light pulse durations: 50, 100 and $2000 \mu \mathrm{s}$. The voltage traces relative to the voltage taps located
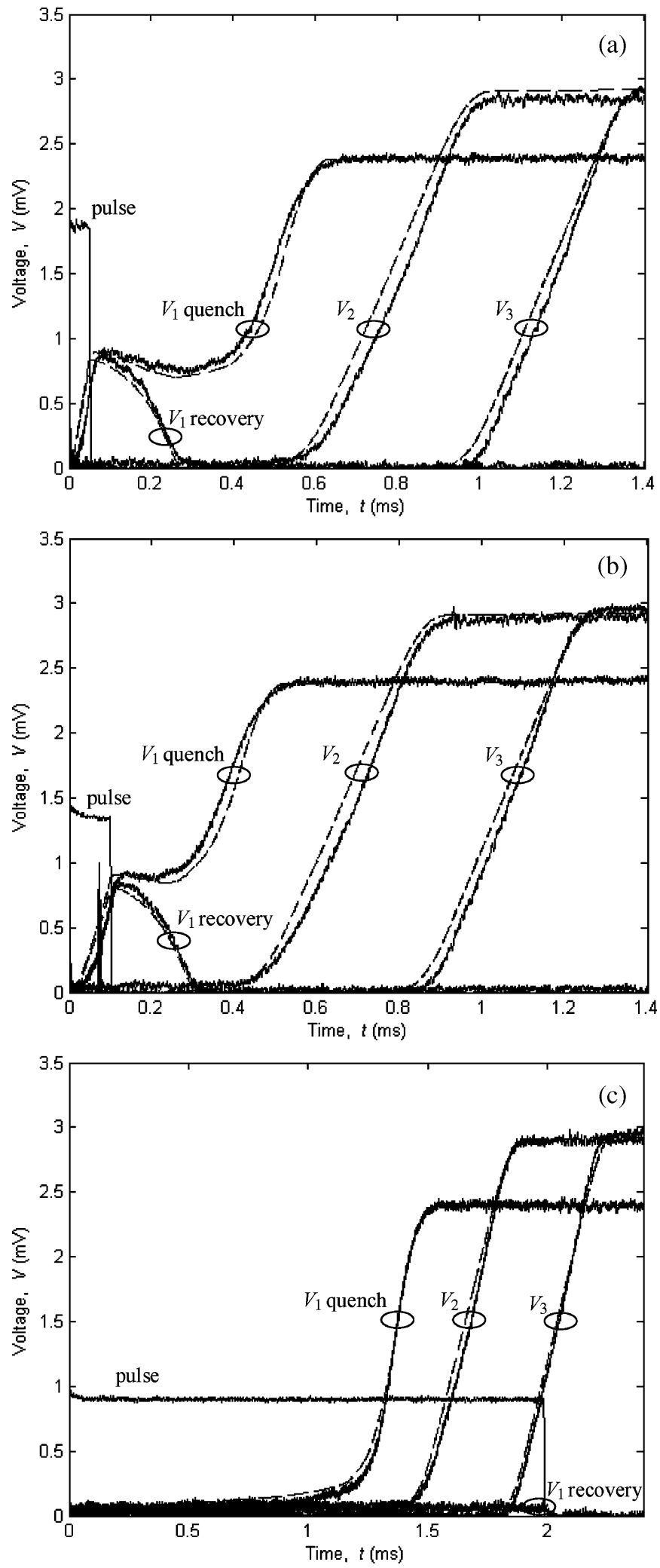

Fig. 2. Measured (solid) and computed (dashed) voltages at $6 \mathrm{~T}, I_{\mathrm{op}}=$ $85 \% I_{\mathrm{C}}, t_{\mathrm{pulse}}=50 \mu \mathrm{s}(\mathrm{a}), 100 \mu \mathrm{s}(\mathrm{b})$ and $2000 \mu \mathrm{s}$ (c).

across the illuminated region $\left(V_{1}\right)$ are reported for both quench and recovery. The curves relative to $V_{2}$ and $V_{3}$ only refer to the quench case. 


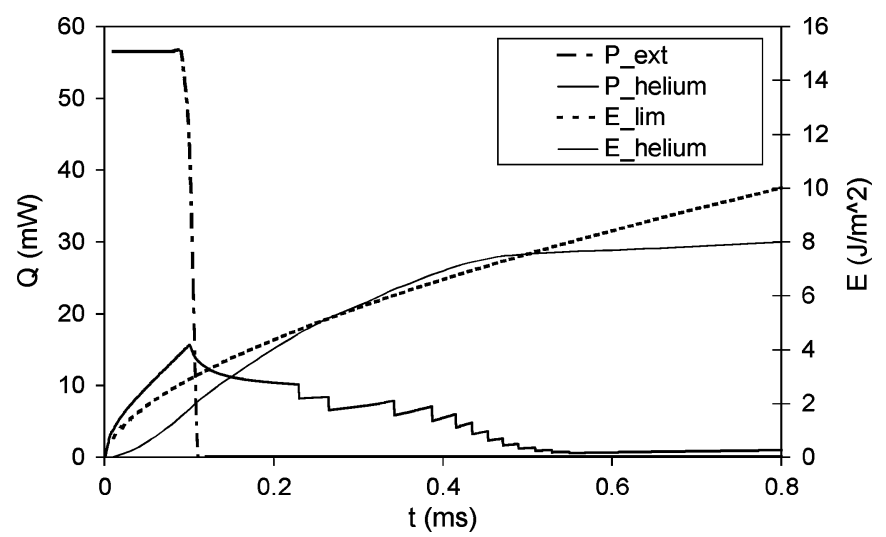

Fig. 3. Power and energy exchanged with the environment by the central wire sector in the quench case reported in Fig. 2(b).

The trace relative to the laser pulse refers to the voltage across the diode laser, and is reported in arbitrary units to show the period of illumination of the wire. The computed and experimental voltage traces show a very good agreement over the whole range of pulse lengths. The model can also reproduce the propagation of quench from the heated region to the rest of the wire, as seen from the $V_{2}$ and $V_{3}$ curves. Similar agreements have been found over a wide range of transport currents and background fields. As a general remark, it is worth noting that in most of the recovery cases that we have analysed, the numerical model does not show any transition at all to film boiling. In a few cases, with low power inputs and long pulse durations $(2 \mathrm{~ms})$, we observe a local transition to film boiling over a small region, which is not able to drive the quench. In all the simulations resulting in quenches, the transition to film boiling spreads over the whole sample. Fig. 3 reports the time evolution of the heat fluxes from the laser and towards the helium for the middle wire sector in the quench case reported in Fig. 2(b). As the total energy transferred to helium reaches the energy limit defined in (3), the heat flux transferred to the helium starts decreasing in successive small steps. These steps correspond to the onset of film boiling in neighboring sectors and result from the smearing described in Section III, which avoids the abrupt variation of heat exchange coefficient that would be obtained with $\sigma=0$.

\section{B. Quench Energy}

The Quench Energies (QE) calculated at $6 \mathrm{~T}$ and $85 \%$ of critical current for different values of light pulse durations are reported in Fig. 4. In the same plot, we also report the value of the total energy emitted by the laser. The computed QE's for long pulses appear to be of the order of 55 to $60 \%$ of the total energy emitted by the laser. This result can be considered as a reasonable estimation of the amount of energy actually absorbed by the wire. The calculated QE's tend toward the total emitted energy for short pulses, which may be too high. In our opinion, this discrepancy could be due to the energetic criterion chosen for the transition from nucleate to film boiling (see (3)). As it turns out, this experimental criterion was established for pulses in the 100-to-1000- $\mu$ s range [6], [9]. Further investigations are

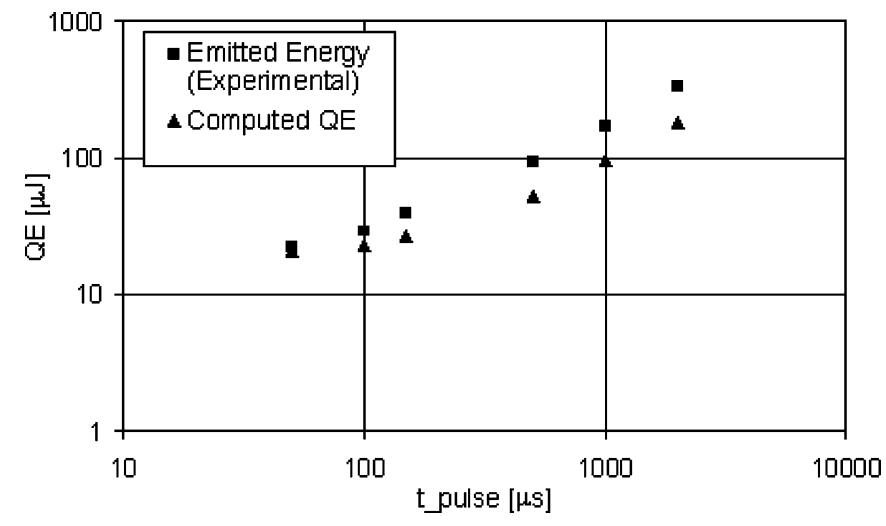

Fig. 4. Measured and computed QE's at $6 \mathrm{~T}$, for $I_{\mathrm{op}}=85 \% I_{\mathrm{C}}$, and different values of $t_{\text {pulse }}$

needed to better understand and describe the phenomena occurring below $100 \mu \mathrm{s}$.

\section{CONCLUSION}

The numerical modeling of the quench and recovery of multifilament composite $\mathrm{Cu} / \mathrm{NbTi}$ wires in pool boiling helium I offers a number of remarkable difficulties. The present study shows that a crucial elements of this modeling is the definition of the transition from nucleate to film boiling. A smearing of the heat exchange coefficient is very useful to better reproduce the experimental results. Another necessary aspect is to allow film-boiling regions to re-enter the nucleate boiling regime at low values of heat flux transferred to the helium. Better estimations of the condition for the onset of film boiling are required for more accurate evaluations of the QE for short pulses.

\section{ACKNOWLEDGMENT}

The authors thank B. Baudouy from CEA Saclay for useful comments and suggestions.

\section{REFERENCES}

[1] K. Seo, M. Morita, S. Nakamura, T. Yamada, and Y. Jizo, "Minimum quench energy measurements for superconducting wires," IEEE Trans. Mag., vol. MAG-32, no. 4, pp. 3089-3093, 1996.

[2] D. E. Baynham, D. A. Cragg, D. C. Coombs, P. Bauer, and R. Wolf, "Transient stability of LHC strands," IEEE Trans. Appl. Supercond., vol. 9, no. 2, pp. 1109-1112, 1999.

[3] F. Trillaud, F. Ayela, M. Fratini, D. Leboeuf, and P. Tixador, "A novel technique for minimum quench energy measurements in superconductors using a single mode diode laser," Cryogenics, vol. 45 , no. 8 , pp. 585-588, 2005.

[4] F. Trillaud, F. Ayela, A. Devred, and P. Tixador, "Investigation of the stability of $\mathrm{Cu} / \mathrm{NbTi}$ multifilament composite wires," IEEE Trans. Appl. Supercond., vol. 16, no. 2, pp. 1712-1716, 2006.

[5] Thea Software. Cryosoft, France, 2003.

[6] C. Schmidt, Review of Steady State and Transient Heat Transfer in Pool Boiling Helium I. Saclay, France, International Institute of Refrigeration: Commission A1/2-Saclay, 1981, pp. 17-31.

[7] M. N. Wilson, Superconducting Magnets. Oxford: Clarendon Press, 1983.

[8] “Handbook of Cryogenic Engineering,” J. G. Weisend, Ed., 1998, Germany.

[9] V. I. Deev, V. S. Kharitonov, K. V. Kutsenko, and A. A. Lavrukhin, "Transient boiling crisis of cryogenic liquids," International Journal of Heat and Mass Transfer, vol. 47, pp. 5477-5482, 2004. 